\title{
Unintegrated dipole gluon distribution at small transverse momentum
}

\author{
Mariyah Siddiqah, Nahid Vasim, Khatiza Banu, and Raktim Abir ${ }^{*}$ \\ Department of Physics, Aligarh Muslim University, Aligarh 202002, India \\ Trambak Bhattacharyya \\ UCT-CERN Research Centre and Department of Physics, $R$ W James Building, \\ University of Cape Town, Rondebosch 7701, Cape Town, South Africa
}

(Received 2 February 2018; published 14 March 2018)

\begin{abstract}
We derive analytical results for unintegrated color dipole gluon distribution function at small transverse momentum. By Fourier transforming the $S$-matrix for large dipoles we derive the results in the form of a series of Bells polynomials. Interestingly, when resumming the series in leading log accuracy, the results showing up striking similarity with the Sudakov form factor with role play of coupling is being done by a constant that stems from the saddle point condition along the saturation line.
\end{abstract}

DOI: $10.1103 /$ PhysRevD.97.054009

\section{INTRODUCTION}

The discovery of rapidly growing cascade of gluons and sharp nonlinear rise of its distributions in Deep inelastic scattering experiments at HERA collider provides indirect experimental evidence that proton at high energy is a hugely complex many body quantum system where gluons are the dominant degrees of freedom. By now many studies have been performed in the theoretical front to develop frameworks that extend our understanding on the structure of proton beyond just one dimensional ordinary parton distribution functions (PDFs). The attempts are mostly based on considering other (than regular PDFs) relatively closer descendants of the original, yet unknown, Wigner distribution functions which presumably contain all the information. Transverse momentum dependent parton distributions (TMDs) or unintegrated parton distribution functions (UPDFs) are such examples that provide, in addition to longitudinal momentum fraction $x$ of the parton, details of transverse momentum distribution and therefore contain much more detailed information on the internal structure of protons relative to the ordinary PDFs [1].

On the experimental front the unintegrated gluon distribution functions are among the key topics to be fully investigated at current and future electron-ion collider facilities including JLab's $12 \mathrm{GeV}$ Upgrade, eRHIC and the planned EIC. The focus would be on both polarized or

\footnotetext{
*raktim.ph@amu.ac.in
}

Published by the American Physical Society under the terms of the Creative Commons Attribution 4.0 International license. Further distribution of this work must maintain attribution to the author(s) and the published article's title, journal citation, and DOI. Funded by SCOAP . unpolarized parton distributions for both spin polarized and unpolarized protons. It has been anticipated that the unintegrated gluon distribution can either be directly probed in the quark-antiquark jet correlation in deep inelastic scattering (for unintegrated Weizsäcker-Williams gluon distributions) or in the direct photon-jet correlation in $p A$ collisions (for unintegrated dipole gluon distributions). The unintegrated gluon and quark distributions involved in other different processes, including other dijet channels in $p A$ collisions, are actually related to this two widely proposed ones: the Weizsäcker-Williams gluon distribution and the dipole gluon distribution in the large- $N_{c}$ limit [2-5].

The deep inelastic scattering experiments at HERA also provide intense indications that there exists a novel, yet unexplored, saturation regime in high energy limit of QCD where the many-body dynamics inside the proton is intrinsically nonlinear in character. In this regime the cascading gluons occupy the phase space in the final state to such an extent that fusion of newly formed gluons begin to start leading to the origin of gluon saturation with a characteristic momentum scale $Q_{s}$ [6]. This fusion of multiple gluons to single gluon eventually restore the unitarity of the scattering $S$-matrix, which will otherwise violated by the almost exponential growth of gluon multiplicity. This was first studied by Balitsky $[7,8]$ within Wilson line formalism leading to a hierarchic chain formed by the Wilson line operators and later by Kovchegov $[9,10]$ in the Mueller's color dipole approach [11-13] where the Balistky hierarchic chain of Wilson line operators reduced to a closed form equation in the large- $N_{c}$ limit. The Balitsky-Kovchegov (BK) equation is an integro-differential equation, the integral kernel for both linear and non linear terms are happen to be identical, and has a simple interpretation of one parent color dipole in the initial state 
splitting into two daughter dipoles in the final state. Since then a lot of progress has been made in solving the BK equation both analytically and numerically and until now only a few limiting analytical solutions of leading order BK equation exists.

In this article we consider Levin-Tuchin (LT) solution $[14,15]$ of the LO BK equation and derive unintegrated dipole gluon distribution, for small transverse momentum, in the form of a series of Bells polynomials. Within the leading $\log$ approximation the series resummed as,

$$
\begin{aligned}
\left.x G^{D P}\left(x, k_{\perp}\right)\right|_{Q_{s} \gtrsim k_{\perp} \gg \Lambda_{\mathrm{QCD}}} \approx & -\frac{S_{\perp} N_{c} \tau}{\pi^{3} \alpha_{s}} \ln \left(\frac{k_{\perp}^{2}}{4 Q_{s}^{2}(Y)}\right) \\
& \times \exp \left[-\tau \ln ^{2}\left(\frac{k_{\perp}^{2}}{4 Q_{s}^{2}(Y)}\right)\right],
\end{aligned}
$$

where $k_{\perp}$ is transverse momentum of the parton, rapidity variable $Y \equiv \ln (1 / x)$ and $\tau \approx 0.2$ a constant that stems from the saddle point condition along the saturation line. As LevinTuchin (LT) solution is valid only for larger dipoles $x_{\perp} \gtrsim$ $1 / Q_{s}$ deep inside the saturation region Eq. (1) is expected to be valid in the relatively smaller momentum range $Q_{s} \gtrsim$ $k_{\perp} \gg \Lambda_{\mathrm{QCD}}$. Employing McLerran-Venugopalan (MV) [16-18] model or phenomenological Golec-Biernat and M. Wusthoff (GBW) [19,20] form of $S$-matrix, we also derive the UDGD function as,

$\left.x G^{D P}\left(x, k_{\perp}\right)\right|_{k_{\perp} \approx Q_{s} \gg \Lambda_{\mathrm{QCD}}} ^{Y \sim 0} \approx \frac{S_{\perp} N_{c}}{2 \pi^{3} \alpha_{s}} \frac{k_{\perp}^{2}}{Q_{s}^{2}(Y)} \exp \left(-\frac{k_{\perp}^{2}}{Q_{s}^{2}(Y)}\right)$,

which is expected to be valid around $Y \sim 0$. We further discussed the distribution in the extended geometric scaling region (just outside the saturation boundary) and also for very large momentum where power-law fall with increasing transverse momentum.

The article is organized as follows: In Sec. II we review the operator definition of unintegrated gluon distribution functions, the WW distribution and color dipole distribution, and their possible connection to $S$-matrix at small $x$. We then adopt form of $S$-matrix valid at different kinematic ranges outside or in the vicinity of saturation boundary. In Sec. III we derived our main result for the unintegrated color dipole gluon distribution at small momentum. Finally we conclude in Sec. IV.

\section{UNINTEGRATED GLUON DISTRIBUTIONS AND SMALL $X$ DYNAMICS}

Both types of universal unintegrated gluon distribution functions: Weizsäcker-Williams (WW) distribution and dipole gluon distribution are essentially dimension four two point correlation function of classical gluon fields (non-abelian Weizsäcker-Williams fields) of relativistic hadrons. The operator definition of Weizsäcker-Williams gluon distribution is,

$$
\begin{aligned}
x G^{W W}\left(x, k_{\perp}\right)= & 2 \int \frac{d \xi^{-} d^{2} \xi_{\perp}}{(2 \pi)^{3} P^{+}} e^{i x P^{+} \xi^{-}-i k_{\perp} \xi_{\perp}} \\
& \times\left\langle P\left|\operatorname{Tr}\left[F^{+i}\left(\xi^{-}, \xi_{\perp}\right) \mathcal{U}^{[+] \dagger} F^{+i}(0,0) \mathcal{U}^{[+]}\right]\right| P\right\rangle,
\end{aligned}
$$

whereas the operator definition of color dipole gluon distribution in the fundamental representation is,

$$
\begin{aligned}
x G^{D P}\left(x, k_{\perp}\right)= & 2 \int \frac{d \xi^{-} d^{2} \xi_{\perp}}{(2 \pi)^{3} P^{+}} e^{i x P^{+} \xi^{-}-i k_{\perp} \cdot \xi_{\perp}} \\
& \times\left\langle P\left|\operatorname{Tr}\left[F^{+i}\left(\xi^{-}, \xi_{\perp}\right) \mathcal{U}^{[-] \dagger} F^{+i}(0,0) \mathcal{U}^{[+]}\right]\right| P\right\rangle .
\end{aligned}
$$

In both the definitions $F^{\mu \nu}$ is gluon field strength tensor $F_{a}^{\mu \nu}$ and the gauge links involved are,

$$
\begin{gathered}
\mathcal{U}^{[+]}=U^{n}\left[0^{-}, 0_{\perp} ; \infty^{-}, 0_{\perp}\right] U^{t}\left[\infty^{-}, 0_{\perp} ; \infty^{-}, \infty_{\perp}\right] \\
\times U^{t}\left[\infty^{-}, \infty_{\perp} ; \infty^{-}, \xi_{\perp}\right] U^{n}\left[\infty^{-}, \xi_{\perp} ; \xi^{-}, \xi_{\perp}\right], \\
\mathcal{U}^{[-]}=U^{n}\left[0^{-}, 0_{\perp} ;-\infty^{-}, 0_{\perp}\right] U^{t}\left[-\infty^{-}, 0_{\perp} ;-\infty^{-}, \infty_{\perp}\right] \\
\times U^{t}\left[-\infty^{-}, \infty_{\perp} ;-\infty^{-}, \xi_{\perp}\right] U^{n}\left[-\infty^{-}, \xi_{\perp} ; \xi^{-}, \xi_{\perp}\right],
\end{gathered}
$$

where the longitudinal $\left(U^{n}\right)$ and transverse $\left(U^{t}\right)$ gauge links are defined as,

$\begin{aligned} U^{n}\left[a^{-}, x_{\perp} ; b^{-}, x_{\perp}\right] & =\mathcal{P} \exp \left[i g \int_{a^{-}}^{b^{-}} d x^{-} A^{+}\left(0, x^{-}, x_{\perp}\right)\right], \\ U^{t}\left[x^{-}, a_{\perp} ; x^{-}, b_{\perp}\right] & =\mathcal{P} \exp \left[i g \int_{a_{\perp}}^{b_{\perp}} d x_{\perp} \cdot A_{\perp}\left(0, x^{-}, x_{\perp}\right)\right] .\end{aligned}$

In some light-cone gauge insertion of transverse gauge links are mandatory in order to maintain the gauge invariance of the distribution functions.

Now one may assume for simplicity that the nucleus is a cylinder with its axis along the $z$-axis, so that saturation momentum $Q_{s}$ does not depend on the impact parameter $\left(b_{\perp}\right)$ (depends however on $x$ ) and integration over $b_{\perp}$ can be carried out simply by multiplying the integrand by the transverse area $\left(S_{\perp}\right)$. It can then be shown that under this assumption, the color dipole gluon distribution can be written as [21],

$$
\begin{aligned}
x G^{D P}\left(x, k_{\perp}\right)= & \frac{S_{\perp} N_{c}}{2 \pi^{2} \alpha_{s}} k_{\perp}^{2} \int \frac{d^{2} r_{\perp}}{(2 \pi)^{2}} e^{-i k_{\perp} \cdot r_{\perp}} \\
& \times \frac{1}{N_{c}}\left\langle\operatorname{Tr} U\left(r_{\perp}\right) U^{\dagger}(0)\right\rangle_{x} \\
= & \frac{S_{\perp} N_{c}}{2 \pi^{2} \alpha_{s}} k_{\perp}^{2} \int \frac{d^{2} r_{\perp}}{(2 \pi)^{2}} e^{-i k_{\perp} \cdot r_{\perp}} S\left(x, r_{\perp}\right),
\end{aligned}
$$


where $S\left(x, r_{\perp}\right)$ is the $S$-matrix for the quark (anti-quark) dipole, with transverse separation of poles being $r_{\perp}$, scattering on a nuclear target with some high energy corresponding to Bjorken scaling variable $x$.

Assumption of color dipoles as the degrees of freedom possibly be the most convenient way to study high energy evolution in QCD. Originally proposed by Mueller, and developed in transverse coordinate space, it is easier to include the saturation effects in the model. In this framework of Mueller dipole model the nonlinear evolution of the $S$-matrix, $S\left(x, r_{\perp}:=x_{\perp}-y_{\perp}\right)$, is governed by the Balitsky-Kovchegov equation (BK), in large- $N_{c}$ limit, as, The BK equation takes into account all long-lived soft gluon emissions, off almost onshell hard quark, with the lifetime of the gluon usually much longer than the size of the nuclear target. In the following we will take different limiting solutions of the above equation in the form of the $S$-matrix to derive $x G^{D P}\left(x, k_{\perp}\right)$ appropriate for corresponding momentum ranges.

\section{A. UDGD outside or in the vicinity of the saturation boundary}

The region with momentum $k_{\perp} \lesssim Q_{s}(Y)$ (corresponding to $r_{\perp} \gtrsim 1 / Q_{s}(Y)$ ), where the nonlinear effects become important, is the saturation region. When the transverse momentum is high enough, $\left(k_{\perp} \gg Q_{s}\right)$, it is well known from $\mathrm{pQCD}$ that $[22,23])$,

$$
\left.x G^{D P}\left(x, k_{\perp}\right)\right|_{k_{\perp} \gg Q_{s}} \propto \frac{1}{k_{\perp}^{2}} .
$$

This is identical for both the unintegrated WeizsäckerWilliams distribution and color dipole distribution.

In the vicinity but just outside of the saturation boundary, $k_{\perp} \gtrsim Q_{s}(Y)$ [corresponding to $r_{\perp} \lesssim 1 / Q_{s}(Y)$ ], dipole amplitude takes the form,

$$
\begin{aligned}
N\left(r_{\perp}, Y\right) & =1-S\left(r_{\perp}, Y\right) \propto\left[r_{\perp} Q_{s}(Y)\right]^{1+2 i \nu_{0}} \\
& \approx\left[r_{\perp}^{2} Q_{s}^{2}(Y)\right]^{\gamma_{\mathrm{cr}}},
\end{aligned}
$$

where $\gamma_{\text {cr }} \sim 0.63$. When substituting back to Eq. (7) UDGD shows expected power law fall in $k_{\perp}$ as [23],

$$
\left.x G^{D P}\left(x, k_{\perp}\right)\right|_{k_{\perp} \gtrsim Q_{s}} \propto \frac{S_{\perp} N_{c}}{2 \pi^{3} \alpha_{s}}\left(\frac{Q_{s}^{2}(Y)}{k_{\perp}^{2}}\right)^{\gamma_{\mathrm{cr}}} .
$$

\section{B. UDGD in GGM, MV, and GBW models}

Kinematic domains where in-medium interactions can be approximated by stochastic multiple soft gluon scatterings the scattering matrix appears to be a Gaussian in dipole size. Outside the saturation region and for small dipole $r_{\perp} \ll 1 / Q_{s}$ the scattering matrix in the Glauber-GribovMueller (GGM) model found to scales with $r_{\perp}$ as,

$S\left(r_{\perp}, Y \sim 0\right)=\exp \left(-\frac{1}{4} r_{\perp}^{2} Q_{s 0}^{2}\right) \approx 1-\frac{1}{4} r_{\perp}^{2} Q_{s 0}^{2}$,

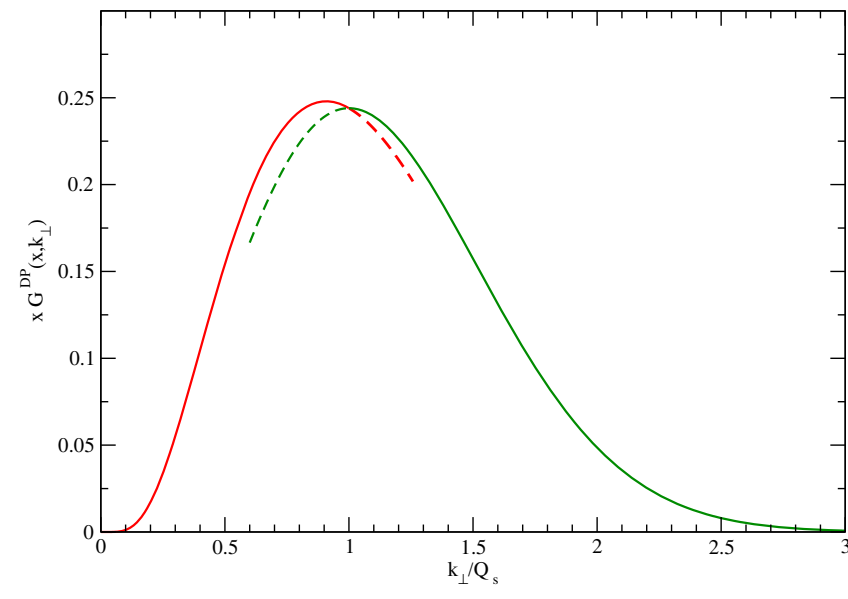

FIG. 1. The unintegrated dipole gluon distribution $x G^{D P}\left(r_{\perp}, Y\right)$ plotted as function of $\xi=k_{\perp} / Q_{s}(Y)$ for nucleus of typical radius $\sim 7 \mathrm{fm}$ at $\alpha_{s} \sim 0.1$. Red line corresponds to Eq. (1) $\left(\propto \ln \xi \exp \left(-\tau \ln ^{2} \xi\right)\right)$ for the small momentum range while green lines corresponds to Eq. (2) $\left(\propto \xi^{2} \exp \left(-\xi^{2}\right)\right)$ for relatively higher momentum. The tail of the green curve, has been tweaked by scaling down to match at $\xi=1$, about to follow power law fall as given in Eq. (10) and Eq. (8).

Since in a zero-size dipole the color charges of quark and the antiquark cancel each other leading to the disappearances of interactions with the target (the color transparency effect), at small $r_{\perp}$ we have $N=1-S \sim r_{\perp}^{2}$, so the amplitude $N$ is zero for zero dipole size. Even in McLerran-Venugopalan model or phenomenological Golec-Biernat and M. Wusthoff model the $S$-matrix takes the following form,

$S_{\mathrm{MV}}\left(r_{\perp}, Y\right)=1-N\left(r_{\perp}, Y\right)=\exp \left(-\kappa r_{\perp}^{2} Q_{s}^{2}(Y)\right)$,

where $N$ is the imaginary part of the dipole-nucleus amplitude and $\kappa \sim 1 / 4$ usually fixed from the definition of the saturation scale $Q_{s}$. Equation (12) is expected to be valid at some low initial rapidity both little inside and outside the saturation region and often used as initial condition for the full $Y$ evolution of $S$-matrix through the Balitsky-Kovchegov equation. The $S$-matrix in both Eq. (11) and Eq. (12) is Gaussian in the variable $x_{\perp} Q_{s}(Y)$ with a (model dependent) variance. Therefore substituting Eq. (12) in Eq. (7) and evaluating the integral is easy leading to a Gaussian $\otimes$ quadratic distribution of transverse momentum as [23,24] (See also [25]),

$$
x G^{D P}\left(x, k_{\perp}\right)=\frac{S_{\perp} N_{c}}{2 \pi^{3} \alpha_{s}} \frac{k_{\perp}^{2}}{Q_{s}^{2}(Y)} \exp \left(-\frac{k_{\perp}^{2}}{Q_{s}^{2}(Y)}\right) .
$$

\section{UDGD DEEP INSIDE THE SATURATION BOUNDARY}

Deep inside saturation region one may take LevinTuchin form of S-matrix valid for larger dipoles 
$x_{\perp} \gtrsim 1 / Q_{s}$ therefore could be appropriate to explore relatively smaller momentum range $Q_{s} \gtrsim k_{\perp} \gg \Lambda_{\mathrm{QCD}}$. Unlike the $\mathrm{S}$-matrix that is Gaussian in $r_{\perp}^{2} Q_{s}^{2}(Y)$, this time it would not be trivial to evaluate the integral as, the Levin-Tuchin solution, having the following expression,

$$
S\left(r_{\perp}, Y\right)=\exp \left(-\tau \ln ^{2}\left[r_{\perp}^{2} Q_{s}^{2}(Y)\right]\right)
$$

${ }^{1}$ is Gaussian in scaling variable, $\ln \left(r_{\perp}^{2} Q_{s}^{2}(Y)\right)$ and valid when it is large $r_{\perp}^{2} Q_{s}^{2}(Y) \gtrsim 1$, leading to the large logarithm in the exponent, and hence, when expanded in series, cannot be truncated at any finite order term in the series, to have a reasonable approximation.

We begin by writing,

$$
\left.x G^{D P}\left(x, k_{\perp}\right)\right|_{Q_{s} \gtrsim k_{\perp} \gg \Lambda_{\mathrm{QCD}}}=\frac{S_{\perp} N_{c}}{2 \pi^{2} \alpha_{s}} k_{\perp}^{2} \int \frac{d^{2} r_{\perp}}{(2 \pi)^{2}} e^{-i k_{\perp} \cdot r_{\perp}} \exp \left(-\tau \ln ^{2}\left[r_{\perp}^{2} Q_{s}^{2}(Y)\right]\right) .
$$

Here we use the identity $\ln ^{2}(y)=\ln ^{2}(1 / y)$, expand the exponential to express it in the form of a series where the $n$th term having $2 n$th derivatives of the dummy variable $\eta$ as,

$$
\begin{aligned}
\left.x G^{D P}\left(x, k_{\perp}\right)\right|_{Q_{s} \gtrsim k_{\perp} \gg \Lambda_{\mathrm{QCD}}} & =\frac{S_{\perp} N_{c}}{2 \pi^{2} \alpha_{s}} k_{\perp}^{2} \sum_{n=0}^{\infty} \frac{(-\tau)^{n}}{n !} \int \frac{d^{2} r_{\perp}}{(2 \pi)^{2}} e^{-i k_{\perp} \cdot r_{\perp}} \ln ^{2 n}\left[\frac{1}{r_{\perp}^{2} Q_{s}^{2}(Y)}\right] \\
& =\frac{S_{\perp} N_{c}}{2 \pi^{2} \alpha_{s}} k_{\perp}^{2} \sum_{n=0}^{\infty} \frac{(-\tau)^{n}}{n !} \lim _{\eta \rightarrow 0} \frac{\partial^{2 n}}{\partial \eta^{2 n}} \int \frac{d^{2} r_{\perp}}{(2 \pi)^{2}} e^{-i k_{\perp} \cdot r_{\perp}}\left[\frac{1}{r_{\perp}^{2} Q_{s}^{2}(Y)}\right]^{\eta}
\end{aligned}
$$

This can further be simplified by changing the order summation and integration as long as the series converges and performing the integration for each term of the series,

$$
\left.x G^{D P}\left(x, k_{\perp}\right)\right|_{Q_{s} \gtrsim k_{\perp} \gg \Lambda_{\mathrm{QCD}}}=\frac{S_{\perp} N_{c}}{2 \pi^{2} \alpha_{s}} k_{\perp}^{2} \sum_{n=0}^{\infty} \frac{(-\tau)^{n}}{n !} \lim _{\eta \rightarrow 0} \frac{\partial^{2 n}}{\partial \eta^{2 n}} \frac{1}{\pi} \frac{d}{d k_{\perp}^{2}}\left(\frac{k_{\perp}^{2}}{4 Q_{s}^{2}(Y)}\right)^{\eta} \frac{\Gamma(1-\eta)}{\Gamma(1+\eta)} .
$$

Besides $2 n$th $\eta$ differentiation Eq. (19) further contains additionally single first order $k_{\perp}^{2}$ derivative. The details of the calculation are in Appendix A. One may now take the $k_{\perp}^{2}$ derivative out of all $\eta$ derivatives and then apply the general Leibniz rule for $n$th derivative of product of two functions to get,

$$
\left.x G^{D P}\left(x, k_{\perp}\right)\right|_{Q_{s} \gtrsim k_{\perp} \gg \Lambda_{\mathrm{QCD}}}=\frac{S_{\perp} N_{c}}{2 \pi^{3} \alpha_{s}} k_{\perp}^{2} \sum_{n=0}^{\infty} \frac{(-\tau)^{n}}{n !} \lim _{\eta \rightarrow 0} \frac{d}{d k_{\perp}^{2}} \sum_{k=0}^{2 n}\left(\begin{array}{c}
2 n \\
k
\end{array}\right)\left[\frac{\partial^{2 n-k}}{\partial \eta^{2 n-k}}\left(\frac{k_{\perp}^{2}}{4 Q_{s}^{2}(Y)}\right)^{\eta}\right] \mathcal{C}_{k}(\eta),
$$

where, for brevity, we define,

$$
\mathcal{C}_{k}(\eta)=\frac{\partial^{k}}{\partial \eta^{k}} \frac{\Gamma(1-\eta)}{\Gamma(1+\eta)}
$$

We first perform the $(2 n-k)$-th order derivatives of $\eta$,

$$
\left.x G^{D P}\left(x, k_{\perp}\right)\right|_{Q_{s} \gtrsim k_{\perp} \gg \Lambda_{\mathrm{QCD}}}=\frac{S_{\perp} N_{c}}{2 \pi^{3} \alpha_{s}} k_{\perp}^{2} \sum_{n=0}^{\infty} \frac{(-\tau)^{n}}{n !} \lim _{\eta \rightarrow 0} \frac{d}{d k_{\perp}^{2}} \sum_{k=0}^{2 n}\left(\begin{array}{c}
2 n \\
k
\end{array}\right) \mathcal{C}_{k}(\eta)\left(\frac{k_{\perp}^{2}}{4 Q_{s}^{2}(Y)}\right)^{\eta} \ln ^{2 n-k}\left(\frac{k_{\perp}^{2}}{4 Q_{s}^{2}(Y)}\right),
$$

${ }^{1}$ with,

$$
\tau=\frac{1+2 i \nu_{0}}{4 \chi\left(0, \nu_{0}\right)}
$$

where the function $\chi$ is defined in terms of the digamma functions,

$$
\chi(0, \nu) \equiv 2 \psi(1)-\psi\left(\frac{1}{2}+i \nu\right)-\psi\left(\frac{1}{2}-i \nu\right) .
$$

Special point $\nu_{0}$ is so chosen that $\nu_{0} \equiv \nu_{s p}\left(r_{\perp}=1 / Q_{s}(Y), Y\right) \approx-0.1275 i$ which leads to $\tau \approx 0.2$. 
then the $k_{\perp}^{2}$ differentiation,

$$
\begin{aligned}
\left.x G^{D P}\left(x, k_{\perp}\right)\right|_{Q_{s} \gtrsim k_{\perp} \gg \Lambda_{\mathrm{QCD}}}= & \frac{S_{\perp} N_{c}}{2 \pi^{3} \alpha_{s}} k_{\perp}^{2} \sum_{n=0}^{\infty} \frac{(-\tau)^{n}}{n !} \lim _{\eta \rightarrow 0} \sum_{k=0}^{2 n}\left(\begin{array}{c}
2 n \\
k
\end{array}\right) \mathcal{C}_{k}(\eta)\left[\left(\frac{\eta}{4 Q_{s}^{2}(Y)}\right)\left(\frac{k_{\perp}^{2}}{4 Q_{s}^{2}(Y)}\right)^{\eta-1} \ln ^{2 n-k}\left(\frac{k_{\perp}^{2}}{4 Q_{s}^{2}(Y)}\right)\right. \\
& \left.+\left(\frac{k_{\perp}^{2}}{4 Q_{s}^{2}(Y)}\right)^{\eta} \frac{d}{d k_{\perp}^{2}} \ln ^{2 n-k}\left(\frac{k_{\perp}^{2}}{4 Q_{S}^{2}(Y)}\right)\right],
\end{aligned}
$$

and finally take the limit $\eta \rightarrow 0$,

$$
x G^{D P}\left(x, k_{\perp}\right)=\frac{S_{\perp} N_{c}}{2 \pi^{3} \alpha_{s}} k_{\perp}^{2} \sum_{n=0}^{\infty} \frac{(-\tau)^{n}}{n !} \sum_{k=0}^{2 n}\left(\begin{array}{c}
2 n \\
k
\end{array}\right) \mathcal{C}_{k}(0) \frac{d}{d k_{\perp}^{2}} \ln ^{2 n-k}\left(\frac{k_{\perp}^{2}}{4 Q_{s}^{2}(Y)}\right) .
$$

Defining, for convenience,

$$
\lim _{\eta \rightarrow 0} \mathcal{C}_{k}(\eta)=\mathcal{C}_{k}(0) \quad \text { and } \quad \beta=\ln \left(\frac{k_{\perp}^{2}}{4 Q_{s}^{2}(Y)}\right)
$$

and noting that,

$$
\beta^{2 n-k}=\frac{(2 n-k) !}{(2 n) !} \frac{\partial^{k}}{\partial \beta^{k}} \beta^{2 n} .
$$

Equation (24) further simplified to,

$$
\left.x G^{D P}\left(x, k_{\perp}\right)\right|_{Q_{s} \gtrsim k_{\perp} \gg \Lambda_{\mathrm{QCD}}}=\frac{S_{\perp} N_{c}}{2 \pi^{3} \alpha_{s}} k_{\perp}^{2} \frac{d}{d k_{\perp}^{2}} \sum_{n=0}^{\infty} \frac{(-\tau)^{n}}{n !} \sum_{k=0}^{2 n} \mathcal{C}_{k}(0) \frac{1}{k !} \frac{\partial^{k}}{\partial \beta^{k}} \beta^{2 n},
$$

We now interchange order of the summations,

$$
\left.x G^{D P}\left(x, k_{\perp}\right)\right|_{Q_{s} \gtrsim k_{\perp} \gg \Lambda_{\mathrm{QCD}}}=\frac{S_{\perp} N_{c}}{2 \pi^{3} \alpha_{s}} k_{\perp}^{2} \frac{d}{d k_{\perp}^{2}} \sum_{k=0}^{\infty} \sum_{n=\left\lceil\frac{k}{2}\right\rceil}^{\infty} \frac{(-\tau)^{n}}{n !} \mathcal{C}_{k}(0) \frac{1}{k !} \frac{\partial^{k}}{\partial \beta^{k}} \beta^{2 n},
$$

Here we note,

$$
\sum_{n=0}^{\left\lceil\frac{k}{2}\right\rceil-1} \frac{(-\tau)^{n}}{n !} \mathcal{C}_{k}(0) \frac{1}{k !} \frac{\partial^{k}}{\partial \beta^{k}} \beta^{2 n}=0
$$

which helps to start the index of both the summations from zero as,

$$
\left.x G^{D P}\left(x, k_{\perp}\right)\right|_{Q_{s} \gtrsim k_{\perp} \gg \Lambda_{\mathrm{QCD}}}=\frac{S_{\perp} N_{c}}{2 \pi^{3} \alpha_{s}} k_{\perp}^{2} \frac{d}{d k_{\perp}^{2}} \sum_{k=0}^{\infty} \mathcal{C}_{k}(0) \frac{1}{k !} \frac{\partial^{k}}{\partial \beta^{k}} \sum_{n=0}^{\infty} \frac{(-\tau)^{n}}{n !} \beta^{2 n},
$$

leading to exponentiation of the second series,

$$
\left.x G^{D P}\left(x, k_{\perp}\right)\right|_{Q_{s} \gtrsim k_{\perp} \gg \Lambda_{\mathrm{QCD}}}=\frac{S_{\perp} N_{c}}{2 \pi^{3} \alpha_{s}} k_{\perp}^{2} \frac{d}{d k_{\perp}^{2}} \sum_{k=0}^{\infty} \mathcal{C}_{k}(0) \frac{1}{k !} \frac{\partial^{k}}{\partial \beta^{k}} \exp \left(-\tau \beta^{2}\right),
$$

which can further be written as,

$$
\left.x G^{D P}\left(x, k_{\perp}\right)\right|_{Q_{s} \gtrsim k_{\perp} \gg \Lambda_{\mathrm{QCD}}}=\frac{S_{\perp} N_{c}}{2 \pi^{3} \alpha_{s}} k_{\perp}^{2} \frac{d}{d k_{\perp}^{2}} \exp \left(-\tau \beta^{2}\right) \sum_{k=0}^{\infty}(-1)^{k} \mathcal{C}_{k}(0) \frac{\tau^{\frac{k}{2}}}{k !} H_{k}(\sqrt{\tau} \beta),
$$


where we use,

$$
H_{k}(x)=(-1)^{k} e^{x^{2}} \frac{d^{k}}{d x^{k}} e^{-x^{2}}
$$

The coefficients $\mathcal{C}_{k}(0)$ remains to evaluate. In Appendix B we have shown in detail that the coefficients $\mathcal{C}_{k}(0)$ can actually be expressed as the Bell's polynomials $[26]^{2}$ of zeta functions for odd integers as,

$$
\mathcal{C}_{k}(0)=B_{k}\left(x_{1}, \ldots, x_{k}\right):=\mathcal{B}_{k}
$$

where,

$$
\begin{aligned}
& x_{1}=2 \gamma, \\
& x_{2}=0, \\
& x_{3}=2(2 !) \zeta(3), \\
& x_{4}=0, \\
& x_{5}=2(4 !) \zeta(5),
\end{aligned}
$$

and $x_{k}=2(k-1) ! \zeta(k)$ if $k$ is odd and $x_{k}=0$ if $k$ even with $\gamma$ being Euler-Mascheroni constant.

$$
\left.x G^{D P}\left(x, k_{\perp}\right)\right|_{Q_{s} \gtrsim k_{\perp} \gg \Lambda_{\mathrm{QCD}}}=\frac{S_{\perp} N_{c}}{2 \pi^{3} \alpha_{s}} k_{\perp}^{2} \frac{d}{d k_{\perp}^{2}} \exp \left(-\tau \beta^{2}\right) \sum_{k=0}^{\infty} \frac{\mathcal{B}_{k}}{k !}(-\sqrt{\tau})^{k} H_{k}(\sqrt{\tau} \beta),
$$

Interestingly, we also note that,

$$
B_{2 n-k}\left[\ln \frac{k_{\perp}^{2}}{4 Q_{s}^{2}(Y)}, 0,0,0,0, \ldots\right]=\ln ^{2 n-k}\left(\frac{k_{\perp}^{2}}{4 Q_{s}^{2}(Y)}\right)
$$

Then, taking the $k_{\perp}^{2}$ derivative out of the summation one may write Eq. (24) as,

$$
\begin{aligned}
\left.x G^{D P}\left(x, k_{\perp}\right)\right|_{Q_{s} \gtrsim k_{\perp} \gg \Lambda_{\mathrm{QCD}}} & \\
& =\frac{S_{\perp} N_{c}}{2 \pi^{3} \alpha_{s}} k_{\perp}^{2} \sum_{n=0}^{\infty} \frac{(-\tau)^{n}}{n !} \frac{d}{d k_{\perp}^{2}} \sum_{k=0}^{2 n}\left(\begin{array}{c}
2 n \\
k
\end{array}\right) B_{k}[2 \gamma, 0,2(2 !) \zeta(3), 0,2(4 !) \zeta(5), 0, \ldots] B_{2 n-k}\left[\ln \frac{k_{\perp}^{2}}{4 Q_{s}^{2}(Y)}, 0,0,0,0, \ldots\right], \\
& =\frac{S_{\perp} N_{c}}{2 \pi^{3} \alpha_{s}} k_{\perp}^{2} \frac{d}{d k_{\perp}^{2}} \sum_{n=0}^{\infty} \frac{(-\tau)^{n}}{n !} B_{2 n}\left[\ln \frac{k_{\perp}^{2}}{4 Q_{s}^{2}(Y)}+2 \gamma, 0,1 ! 2 \zeta(3), 0,4 ! 2 \zeta(5), \ldots,(2 n-2) ! 2 \zeta(2 n-1), 0\right] .
\end{aligned}
$$

\section{A. Leading log resummation}

For $k_{\perp}^{2} \ll Q_{s}^{2}$ one may approximate the Bell's polynomials as,

$$
B_{2 n}\left[\ln \frac{k_{\perp}^{2}}{4 Q_{s}^{2}(Y)}+2 \gamma, 0,1 ! 2 \zeta(3), 0,4 ! 2 \zeta(5), . .,(2 n-2) ! 2 \zeta(2 n-1), 0\right] \approx\left(\ln \frac{k_{\perp}^{2}}{4 Q_{s}^{2}(Y)}+2 \gamma\right)^{2 n}
$$

and the unintegrated gluon distribution can be approximated (to the leading log) as:

$$
\left.x G^{D P}\left(x, k_{\perp}\right)\right|_{Q_{s} \gg k_{\perp} \gg \Lambda_{\mathrm{QCD}}}=\frac{S_{\perp} N_{c}}{2 \pi^{3} \alpha_{s}} k_{\perp}^{2} \frac{d}{d k_{\perp}^{2}} \exp \left[-\tau\left(\ln \left(\frac{k_{\perp}^{2}}{4 Q_{s}^{2}}\right)+2 \gamma\right)^{2}\right],
$$

\footnotetext{
${ }^{2}$ The complete exponential Bell polynomial $B_{n}\left(x_{1}, \ldots, x_{n}\right)$ is defined by,

$$
\exp \left(\sum_{j=1}^{\infty} x_{j} \frac{t^{j}}{j !}\right)=\sum_{n=0}^{\infty} B_{n}\left(x_{1}, \ldots, x_{n}\right) \frac{t^{n}}{n !}
$$

The Bell's polynomial appear in the study of set partitions. The $n$th Bell's polynomial is defined by $B_{n}\left(x_{1}, x_{2}, \ldots \ldots x_{n}\right)=$ $\sum_{k=1}^{n} B_{n, k}\left(x_{1}, x_{2}, \ldots \ldots x_{n-k+1}\right)$, where $B_{n, k}$ are the partial Bell's polynomials. Let's take an example: for $n=3$, $k=2$, $B_{3,2}\left(x_{1}, x_{2}\right)=3 x_{1} x_{2} . B_{3,2}$ tells us that we are considering partitioning a set of 3 elements into two boxes. $x_{i}$ indicates a box with $i$ elements, and the coefficient in front indicates the number of ways partitioning can be done.
} 


$$
\begin{aligned}
& =-\frac{S_{\perp} N_{c} \tau}{\pi^{3} \alpha_{s}}\left[\ln \left(\frac{k_{\perp}^{2}}{4 Q_{s}^{2}}\right)+2 \gamma\right] \exp \left[-\tau\left(\ln \left(\frac{k_{\perp}^{2}}{4 Q_{s}^{2}}\right)+2 \gamma\right)^{2}\right], \\
& \approx-\frac{S_{\perp} N_{c} \tau}{\pi^{3} \alpha_{s}} \ln \left(\frac{k_{\perp}^{2}}{4 Q_{s}^{2}}\right) \exp \left[-\tau \ln ^{2}\left(\frac{k_{\perp}^{2}}{4 Q_{s}^{2}}\right)\right] .
\end{aligned}
$$

Inside the saturation region, the dipole gluon distribution is expected to go to zero as $k_{\perp}^{2} \rightarrow 0$. This is indeed the case here, however, interestingly, we observe a Sudakov double logarithm type factor in the argument of the exponential function and also, at small transverse momentum, $x G^{D P}\left(x, k_{\perp}\right)$ not proportional to $k_{\perp}^{2}$, as previously anticipated [3,22], rather it is proportional to $\ln \left(k_{\perp}^{2} / 4 Q_{s}^{2}\right)$ times the double log soft factor. In Fig. [1] we have plotted unintegrated dipole gluon distribution (UDGD) both inside and outside the saturation boundary. While UDGD follows Eq. (13) in the proximity of the boundary, deep inside the saturation boundary, where the momentum is relatively small, it follows Eq. (40). We note here until Eq. (36) we are carrying the $k_{\perp}^{2}$ (and also one $d / d k_{\perp}^{2}$, origin of which can be traced back to Appendix A). The $k_{\perp}^{2}$ is been killed when we perform the summation in Eq. (36), in leading log approximation, and then operate the derivative, to reach our final result. After operating the $d / d k_{\perp}^{2}$ on the exponential it generates one $1 / k_{\perp}^{2}$ that kills the $k_{\perp}^{2}$ in the numerator. This indicates that evolution kills the $k_{\perp}^{2}$ behavior from Eq. (2) to Eq. (1) in the small momentum limit and modifies it to be a $\log \otimes \log$ normal distribution as given in Eq. (1) which is the main finding of this work.

\section{CONCLUSION AND OUTLOOK}

Classical asymptotic analysis covers the study of the limiting behavior of functions when some special (singular or non singular) points are approached. Asymptotic expansions usually give increasingly better approximations as the special points are approached, yet not necessarily they always converge to the actual function. In this paper we derive results for unintegrated color dipole gluon distribution function at small transverse momentum. By Fourier transforming the $S$ matrix for large dipoles we derive the results in the form of a series of Bells polynomials that comes in the study of combinatorics. Interestingly, when resumming the series in leading log accuracy, the results showing up striking similarity with the Sudakov form factor with role play of coupling is being done by a constant that stems from the saddle point condition along the saturation line. In QCD nonperturbative effects, within the framework of perturbative analysis, stems from the asymptotic nature of the perturbation series having coupling as the expansion parameter. In this context it would be interesting to see how nonperturbative renormalon contribution shows up when including running coupling effects in this study. It would be also interesting to see how this connects with Collins-Soper-Sterman evolution of TMDs [27,28]. As $S$-matrix directly connects with the probability of transverse deflection of the interacting parton this result could therefore possibly be useful to study medium sensitive observables such as jet quenching parameter [25]. We are also working on to see how the result modify when taking, the recently derived analytical, solution that valid both deep in and way out of the saturation region [29] and why leading log approximation of the solution posses similarity with the Sudakov soft factor [30-32].

\section{ACKNOWLEDGMENTS}

We indebted to Yuri Kovchegov for important suggestions on this work. We also thank Bo-Wen Xiao, Kirill Tuchin, Cyrille Marquet for valuable comments on the draft. This work was supported in part by the University Grants Commission under UGC-BRS Research Start-UpGrant Grant No. F.30-310/2016(BSR). T. B. acknowledges the University Research Committee, University of Cape Town, South Africa for support.

\section{APPENDIX A: INTEGRAL FOR THE HANKEL TRANSFORM}

For circularly symmetric function two dimensional Fourier transform is essentially the Hankel transform of order zero,

$$
\begin{aligned}
I & :=\int \frac{d^{2} r_{\perp}}{(2 \pi)^{2}} e^{-i k_{\perp} \cdot r_{\perp}}\left[\frac{1}{r_{\perp}^{2} Q_{s}^{2}(Y)}\right]^{\eta} \\
& =\frac{1}{2 \pi} Q_{s}^{-2 \eta}(Y) \int_{0}^{\infty} d r_{\perp} r_{\perp} J_{0}\left(r_{\perp} k_{\perp}\right) r_{\perp}^{-2 \eta} .
\end{aligned}
$$

The integral in Eq. (A1) can be evaluated using following integral identities of Bessels functions,

$$
\int_{0}^{\infty} d k k^{\lambda-1} J_{0}(k x)=2^{\lambda-1} x^{-\lambda} \frac{\Gamma(\lambda / 2)}{\Gamma\left(1-\frac{\lambda}{2}\right)} .
$$

Therefore,

$$
I=\frac{\eta}{\pi}\left(\frac{1}{4 Q_{s}^{2}(Y)}\right)^{\eta} \frac{\Gamma(1-\eta)}{\Gamma(1+\eta)}\left(k_{\perp}^{2}\right)^{\eta-1},
$$

which may then further be simplified to,

$$
I=\frac{1}{\pi} \frac{d}{d k_{\perp}^{2}}\left(\frac{k_{\perp}^{2}}{4 Q_{s}^{2}(Y)}\right)^{\eta} \frac{\Gamma(1-\eta)}{\Gamma(1+\eta)} .
$$


Unintegrated dipole gluon distribution can then be written as,

$$
\begin{aligned}
x G^{D P}\left(Y \equiv \ln \frac{1}{x}, k_{\perp}\right) \mid= & \frac{S_{\perp} N_{c}}{2 \pi^{2} \alpha_{s}} k_{\perp}^{2} \sum_{n=0}^{\infty} \frac{(-\tau)^{n}}{n !} \lim _{\eta \rightarrow 0} \frac{\partial^{2 n}}{\partial \eta^{2 n}} \frac{1}{\pi} \frac{d}{d k_{\perp}^{2}} \\
& \times\left(\frac{k_{\perp}^{2}}{4 Q_{s}^{2}(Y)}\right)^{\eta} \frac{\Gamma(1-\eta)}{\Gamma(1+\eta)} .
\end{aligned}
$$

\section{APPENDIX B: EVALUATION OF $\mathcal{C}_{\boldsymbol{k}}$}

When $|z|<1$ the function $\Gamma(1+z)$ can be written as

$$
\Gamma(1+z)=\exp \left(-\gamma z+\sum_{j=2}^{\infty}(-1)^{j} \zeta(j) \frac{z^{j}}{j}\right)
$$

Therefore,

$\frac{\Gamma(1-\eta)}{\Gamma(1+\eta)}=\exp \left(2 \gamma \eta+2 \sum_{j=1}^{\infty} \zeta(2 j+1) \frac{\eta^{2 j+1}}{2 j+1}\right)$

Hence the coefficients $\mathcal{C}_{k}$ could be written as Bell's polynomials for Riemann zeta function at odd integers as,

$$
\mathcal{C}_{k}=B\left(x_{1}, x_{2}, x_{3}, \ldots, x_{j}, \ldots, x_{k}\right)
$$

where $x_{1}=2 \gamma$ and $x_{j}=2(j-1) ! \zeta(j)$ if $j$ is odd integer and zero if even. Initial a few coefficients are as follows,

$$
\begin{aligned}
& \mathcal{C}_{0}(0)=1 \\
& \mathcal{C}_{1}(0)=B[2 \gamma]=2 \gamma \\
& \mathcal{C}_{2}(0)=B[2 \gamma, 0]=(2 \gamma)^{2} \\
& \mathcal{C}_{3}(0)=B[2 \gamma, 0,2(2 !) \zeta(3)]=(2 \gamma)^{3}-2 \psi(2,1), \\
& \mathcal{C}_{4}(0)=B[2 \gamma, 0,2(2 !) \zeta(3), 0]=(2 \gamma)^{4}-16 \gamma \psi(2,1), \\
& \mathcal{C}_{5}(0)=B[2 \gamma, 0,2(2 !) \zeta(3), 0,2(4 !) \zeta(5)]=(2 \gamma)^{5}-80 \gamma^{2} \psi(2,1)-2 \psi(4,1),
\end{aligned}
$$

where $\psi$ is polygamma function.

[1] A. Bacchetta, Where do we stand with a 3-D picture of the proton?, Eur. Phys. J. A 52, 163 (2016).

[2] F. Dominguez, C. Marquet, B. W. Xiao, and F. Yuan, Universality of unintegrated gluon distributions at small x, Phys. Rev. D 83, 105005 (2011).

[3] M. G. A. Buffing, P. J. Mulders, and A. Mukherjee, Universality of quark and gluon TMD correlators, Int. J. Mod. Phys. Conf. Ser. 25, 1460003 (2014).

[4] Y. Hatta, B. W. Xiao, and F. Yuan, Probing the Small-x Gluon Tomography in Correlated Hard Diffractive Dijet Production in Deep Inelastic Scattering, Phys. Rev. Lett. 116, 202301 (2016).

[5] B. W. Xiao, F. Yuan, and J. Zhou, Transverse momentum dependent parton distributions at small-x, Nucl. Phys. B921, 104 (2017).

[6] L. V. Gribov, E. M. Levin, and M. G. Ryskin, Semihard processes in QCD, Phys. Rep. 100, 1 (1983).

[7] I. Balitsky, Operator expansion for high-energy scattering, Nucl. Phys. B463, 99 (1996).

[8] I. Balitsky, in At the Frontier of Particle Physics, edited by M. Shifman, Vol. 2 (World Scientific, Singapore, 2001), p. 1237.
[9] Y. V. Kovchegov, Small $x F_{2}$ structure function of a nucleus including multiple pomeron exchanges, Phys. Rev. D 60, 034008 (1999).

[10] Y. V. Kovchegov, Unitarization of the BFKL pomeron on a nucleus, Phys. Rev. D 61, 074018 (2000).

[11] A. H. Mueller, Soft gluons in the infinite momentum wave function and the BFKL pomeron, Nucl. Phys. B415, 373 (1994).

[12] A. H. Mueller and B. Patel, Single and double BFKL pomeron exchange and a dipole picture of high-energy hard processes, Nucl. Phys. B425, 471 (1994).

[13] Z. Chen and A. H. Mueller, The dipole picture of highenergy scattering, the BFKL equation and many gluon compound states, Nucl. Phys. B451, 579 (1995).

[14] E. Levin and K. Tuchin, Solution to the evolution equation for high parton density QCD, Nucl. Phys. B573, 833 (2000).

[15] E. Levin and K. Tuchin, New scaling at high-energy DIS, Nucl. Phys. A691, 779 (2001).

[16] L. D. McLerran and R. Venugopalan, Computing quark and gluon distribution functions for very large nuclei, Phys. Rev. D 49, 2233 (1994). 
[17] L. D. McLerran and R. Venugopalan, Gluon distribution functions for very large nuclei at small transverse momentum, Phys. Rev. D 49, 3352 (1994).

[18] L. D. McLerran and R. Venugopalan, Green's functions in the color field of a large nucleus, Phys. Rev. D 50, 2225 (1994).

[19] K. J. Golec-Biernat and M. Wusthoff, Saturation in diffractive deep inelastic scattering, Phys. Rev. D 60, 114023 (1999).

[20] K. J. Golec-Biernat and M. Wusthoff, Saturation effects in deep inelastic scattering at low $Q^{2}$ and its implications on diffraction, Phys. Rev. D 59, 014017 (1998).

[21] J. Jalilian-Marian, A. Kovner, L. D. McLerran, and H. Weigert, The intrinsic glue distribution at very small $\mathrm{x}$, Phys. Rev. D 55, 5414 (1997).

[22] D. Kharzeev, Y. V. Kovchegov, and K. Tuchin, Cronin effect and high $p_{T}$ suppression in pA collisions, Phys. Rev. D 68, 094013 (2003).

[23] C. Marquet, E. Petreska, and C. Roiesnel, Transversemomentum-dependent gluon distributions from JIMWLK evolution, J. High Energy Phys. 10 (2016) 065.

[24] E. Petreska, Forward di-jet production in dilute-dense collisions, Nucl. Phys. A956, 894 (2016).

[25] R. Abir, Small- $x$ evolution of jet quenching parameter, Phys. Lett. B 748, 467 (2015).
[26] E. T. Bell, Partition polynomials, Ann. Math. 29, 38 (1927).

[27] J. C. Collins and D. E. Soper, Back-to-back jets in QCD, Nucl. Phys. B193, 381 (1981); Erratum, Nucl. Phys. B213, 545 (1983).

[28] J. C. Collins, D. E. Soper, and G. F. Sterman, Transverse momentum distribution in Drell-Yan pair and $\mathrm{W}$ and $\mathrm{Z}$ boson production, Nucl. Phys. B250, 199 (1985).

[29] R. Abir and M. Siddiqah, Solution of linearized Balitsky-Kovchegov equation, Phys. Rev. D 95, 074035 (2017).

[30] T. Liu and A. A. Penin, High-Energy Limit of QCD beyond Sudakov Approximation, Phys. Rev. Lett. 119, 262001 (2017).

[31] A. A. Penin, High-energy limit of quantum electrodynamics beyond Sudakov approximation, Phys. Lett. B 745, 69 (2015); Erratum, Phys. Lett. B 751, 596(E) (2015); Erratum, Phys. Lett. B 771, 633(E) (2017).

[32] A. H. Mueller, B. W. Xiao, and F. Yuan, Sudakov Resummation in Small- $x$ Saturation Formalism, Phys. Rev. Lett. 110, 082301 (2013). 